\title{
Proportional-Type Output Voltage-Tracking Controller for Interleaved DC/DC Boost Converter with Performance Recovery Property
}

\author{
Seok-Kyoon Kim \\ Department of Creative Convergence Engineering, Hanbat National University, Daejeon 341-58, Republic of Korea \\ Correspondence should be addressed to Seok-Kyoon Kim; lotus45kr@gmail.com
}

Received 7 August 2017; Accepted 18 February 2018; Published 19 March 2018

Academic Editor: Bruno G. M. Robert

Copyright (c) 2018 Seok-Kyoon Kim. This is an open access article distributed under the Creative Commons Attribution License, which permits unrestricted use, distribution, and reproduction in any medium, provided the original work is properly cited.

\begin{abstract}
This paper proposes an offset-free proportional-type output voltage-tracking algorithm embedding the disturbance observers (DOBs) for the $\mathrm{N}$-phase interleaved DC/DC boost converter through a systematical multivariable approach. The contributions of this article fall into two parts. The first one is to design the first-order nonlinear DOBs for exponentially estimating the disturbances caused by the model-plant mismatches. The second one is to prove that the proposed proportional-type controller equipped with the DOBs guarantees the performance recovery property as well as the offset-free property. The performance of the proposed method is evaluated through simulations and experiments using a $3-\mathrm{kW}$ four-phase interleaved DC/DC boost converter, comparing the proposed and feedback linearizing (FL) methods.
\end{abstract}

\section{Introduction}

The DC/DC converters have been widely utilized to supply a high quality DC power despite the disturbances for a variety of industrial applications such as uninterruptible power supply and solar photovoltaic systems [1-6]. In these applications, the DC/DC converters are required to be controlled their current and output voltage with a satisfactory closed-loop performance for a wide range of operating region in the presence of the converter parameter and unexpected load variations.

The cascade output voltage control strategy [7] has been commonly adopted to adjust the output voltage of the DC/DC converters where the inner and outer-loop controllers were designed to control the current and output voltage in a cascade manner. The proportional-integral (PI) controller equipped with nonlinearity cancellation terms has been mainly used for implementing both inner-loop and outerloop controllers $[7,8]$, which can be interpreted as an feedback linearizing (FL) method in the control theoretical point of view. The corresponding control gains were determined for the resulting closed-loop error dynamics to be the desired low-pass filter (LPF) behavior using the converter parameters such as inductance and capacitance values. However, it is questionable that their closed-loop performance would be remaining satisfactory for the various load conditions despite the parameter uncertainties.

There have been many alternative solutions based on the novel control strategy for the inner loop to attain a better closed-loop performance, which includes the deadbeat controllers [9], predictive controllers [10], sliding mode controllers [11], adaptive controllers [12], model predictive controllers [13], and robust controllers [14]. Aside from the adaptive technique, however, these novel schemes require using the converter true parameter values so as to guarantee the closed-loop stability and performance. The adaptive and sliding mode schemes were built using the true inductance value that can be dramatically decreased with increasing the inductor current. The disturbance observer (DOB) based methods were proposed for the robot manipulation [15] and the DC motor control applications [16]. Unlike [16], it is rigorously proved that the proposed proportional-type controller with the DOBs guarantees to not only exponentially recover the target output voltage-tracking performance, but 


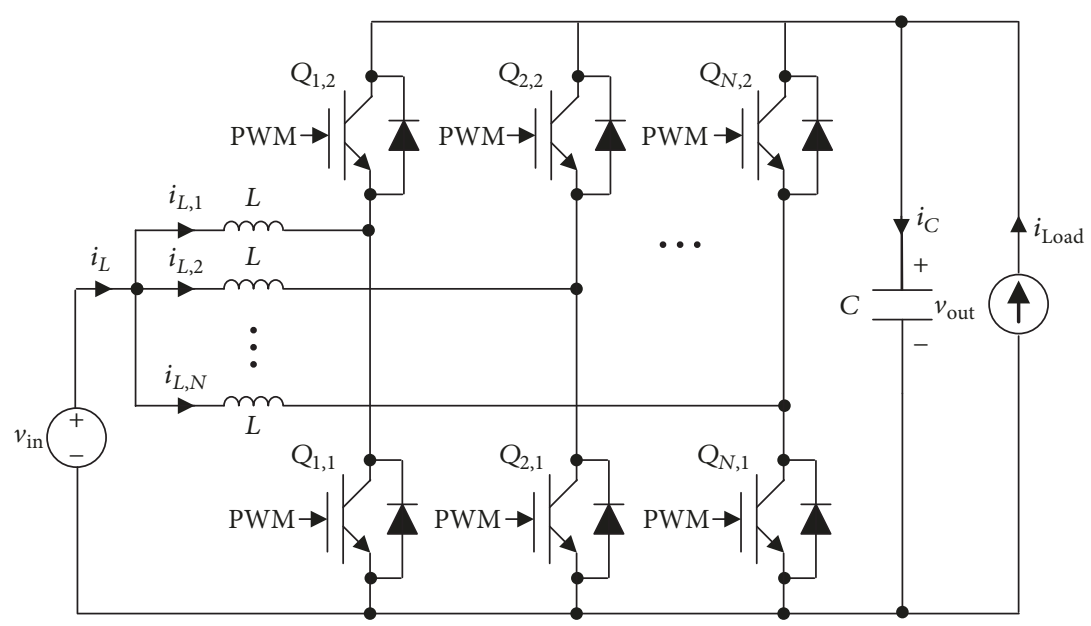

Figure 1: Interleaved DC/DC boost converter circuit.

also remove the steady state errors, which means that the tracking error integrators can be eliminated as well as the corresponding antiwindup algorithms.

This article presents a robust output voltage-tracking algorithm based on a systematical multi-variable approach without tracking error integrators for the $N$-phase interleaved DC/DC boost converter, considering a planet-model mismatch such as parameter and load variations. The novelties of the proposed method are twofold; the exponentially convergent DOBs are constructed for the current and output voltage loops, and the proportional-type nonlinear controller is developed with the exponential performance recovery property and the proof of the offset-free property. These two novelties simplify the implementations of the control algorithm by eliminating the use of tracking error integrators with the anti-windup parts as well as the gain scheduling algorithms, which is the sharp contrast to the above mentioned existent methods such as model predictive and adaptive methods. The simulations and experimental results using a $3-\mathrm{kW}$ four-phase interleaved DC/DC boost converter show the effectiveness of the proposed method, comparing it with the FL method.

\section{Averaged Dynamics of Interleaved DC/DC Boost Converter}

This section briefly describes the dynamical equations of the synchronous-type $N$-phase interleaved DC/DC boost converter depicted in Figure 1, where the duty ratio of $D_{i} \in$ $[0,1], i=1,2, \ldots, N$, is considered as the control action to be applied, and the phase inductor current of $i_{L, i}(t), i=$ $1,2, \ldots, N$, and the capacitor voltage of $v_{\text {out }}(t)$ are treated as the state variables. Unlike the traditional single-phase boost converter, a low voltage drop switch is used as the upper-side diode and is alternatively operated with a lowerside switch, and this type of converter never enters the discontinuous conduction mode (DCM) for all time [17]. Note that the duty ratio of $D_{i}, i=1,2, \ldots, N$, produces the switching command by performing the pulse width modulation (PWM) operation. For example, the duty ratio of $D_{i} \in(0,1)$ renders the switch to be turned on for a period $D_{i} T_{s}$ and the switch to be turned off for a period $\left(1-D_{i}\right) T_{s}$, with $T_{s}$ denoting the PWM period. Due to these operations, the averaged converter dynamics are obtained as

$$
\begin{aligned}
L \frac{d i_{L, i}(t)}{d t}=-\left(1-u_{i}(t)\right) v_{\mathrm{dc}}(t)+v_{\mathrm{in}}, & \\
& i=1,2, \ldots, N, \\
C \frac{d v_{\mathrm{dc}}(t)}{d t}=\sum_{i=1}^{N}\left(1-u_{i}(t)\right) i_{L, i}(t)-i_{\text {Load }}(t), &
\end{aligned}
$$

$\forall t \geq 0$, where the input source voltage is given as $v_{\text {in }}$, $u_{i}(t):=D_{i} \in(0,1)$ and $i_{\text {Load }}(t)$ denotes the load current. See $[6,7,18,19]$ for a detailed derivation of the averaged dynamical equations of (1).

Due to an abrupt load current variation and the parameter uncertainties, such as inductance and capacitance values, the converter dynamics of (1) are rewritten regarding to the nominal parameters as

$$
\begin{aligned}
& L_{0} \frac{d i_{L, i}(t)}{d t}=-\left(1-u_{i}(t)\right) v_{\mathrm{dc}}(t)+v_{\mathrm{in}, 0}+w_{L, i, o}(t), \\
& i=1,2, \ldots, N, \\
& C_{0} \frac{d v_{\mathrm{dc}}(t)}{d t}=\sum_{i=1}^{N}\left(1-u_{i}(t)\right) i_{L, i}(t)+w_{v, o}(t),
\end{aligned}
$$

$\forall t \geq 0$, with the nominal inductance and capacitance values of $L_{0}, C_{0}$, and the initial input voltage $v_{\mathrm{in}, 0}$, where $w_{L, i, o}(t)$, $w_{v, o}(t)$ denote the unknown lumped disturbances caused from the parameter mismatch, unmodelled dynamics, and load uncertainties.

\section{Output Voltage Controller Design}

Let $V_{\mathrm{dc}}^{*}(s)$ and $V_{\mathrm{dc}, \text { ref }}(s)$ be the Laplace transforms of desired output voltage behavior $v_{\mathrm{dc}}^{*}(t)$ and its reference $v_{\mathrm{dc}, \text { ref }}(t)$, 
respectively. The objective of this section is to construct the control law for rendering the closed-loop output voltage dynamics to be

$$
\frac{V_{\mathrm{dc}}^{*}(s)}{V_{\mathrm{dc}, \mathrm{ref}}(s)}=\frac{\omega_{\mathrm{vc}}}{s+\omega_{\mathrm{vc}}}, \quad \forall s \in \mathbb{C},
$$

with $\omega_{\mathrm{vc}}$ being the assignable cut-off frequency as a design parameter, while guaranteeing the current-tracking property:

$$
\lim _{t \rightarrow \infty} i_{L, i}(t)=i_{L, i, \text { ref }}(t), \quad i=1, \ldots, N .
$$

In order to achieve the control objective of (4), consider the time domain expression of the target dynamics of (4) given by

$$
\dot{v}_{\mathrm{dc}}^{*}(t)=\omega_{\mathrm{vc}}\left(v_{\mathrm{dc}, \text { ref }}(t)-v_{\mathrm{dc}}^{*}(t)\right), \quad \forall t \geq 0,
$$

and define the output voltage-tracking error as $\widetilde{v}_{\mathrm{dc}}^{*}(t):=$ $v_{\mathrm{dc}}^{*}(t)-v_{\mathrm{dc}}(t), \forall t \geq 0$, which gives the output voltage-tracking error dynamics using (3):

$$
\begin{aligned}
C_{0} \dot{\tilde{v}}_{\mathrm{dc}}^{*}(t)= & C_{0} \dot{v}_{\mathrm{dc}}^{*}(t)-C_{0} \dot{v}_{\mathrm{dc}}(t) \\
= & -\sum_{i=1}^{N}\left(1-u_{i}(t)\right) i_{L, i}(t)+w_{v}(t) \\
= & -\sum_{i=1}^{N}\left(1-u_{i}(t)\right) i_{L, i, \mathrm{ref}}(t) \\
& +\sum_{i=1}^{N}\left(1-u_{i}(t)\right) \tilde{i}_{L, i}(t)+w_{v}(t), \quad \forall t \geq 0,
\end{aligned}
$$

where $w_{v}(t):=C_{0} \dot{v}_{\mathrm{dc}}^{*}(t)-w_{v, o}(t), \tilde{i}_{L, i}(t):=i_{L, i, \text { ref }}(t)-i_{L, i}(t)$, $\forall t \geq 0$. An inductor current reference signal is proposed as a stabilizing solution to the output voltage-tracking error dynamics of (7):

$$
i_{L, i, \text { ref }}(t)=\frac{1}{N} \frac{1}{\left(1-u_{i}(t)\right)}\left(C_{0} \lambda_{v} \widetilde{v}_{\mathrm{dc}}^{*}(t)+\widehat{w}_{v}(t)\right),
$$

$i=1, \ldots, N, \forall t \geq 0$, where $\lambda_{v}>0$ denotes a tuning parameter; the estimated disturbance $\widehat{w}_{v}(t)$ is given by

$$
\widehat{w}_{v}(t):=\zeta_{v}(t)+l_{v} C_{0} \widetilde{v}_{\mathrm{dc}}^{*}(t), \quad \forall t \geq 0,
$$

and $\zeta_{v}(t)$ stands for the state of the nonlinear observer:

$$
\begin{aligned}
\dot{\zeta}_{v}(t)= & -l_{v} \zeta_{v}(t)-l_{v}^{2} C_{0} \tilde{v}_{\mathrm{dc}}^{*}(t) \\
& +l_{v} \sum_{i=1}^{N}\left(1-u_{i}(t)\right) i_{L, i}(t), \quad \forall t \geq 0,
\end{aligned}
$$

with $l_{v}>0$ being the observer gain. For the rest of this article, the combination of two equations of (10) and (9) is called the output voltage disturbance observer (DOB).

The substitution of the inductance current reference of (8) to the output voltage-tracking error dynamics of (7) leads to

$$
\begin{aligned}
C_{0} \dot{\tilde{v}}_{\mathrm{dc}}^{*}(t)= & -C_{0} \lambda_{v} \widetilde{v}_{\mathrm{dc}}^{*}(t)+\Delta w_{v}(t) \\
& +\sum_{i=1}^{N}\left(1-u_{i}(t)\right) \tilde{i}_{L, i}(t), \quad \forall t \geq 0,
\end{aligned}
$$

where $\Delta w_{v}(t):=w_{v}(t)-\widehat{w}_{v}(t), \forall t \geq 0$. Lemma 1 derives the closed-loop property of (11), which is used for analyzing the whole closed-loop stability analysis.

Lemma 1. Assuming that the disturbance of $w_{v}(t)$ converges to its steady state value $w_{v}^{0}$, exponentially, that is, there is a positive constant of $k_{v}>0$ such that

$$
\dot{\tilde{w}}_{v}^{0}(t)=-k_{v} \widetilde{w}_{v}^{0}(t), \quad \forall t>0
$$

where $\widetilde{w}_{v}^{0}(t):=w_{v}^{0}-w_{v}(t), \forall t \geq 0$, then, a specified design parameter of $\lambda_{v}>0$ with the DOB gains of

$$
l_{v}=\frac{3}{4 C_{0} \lambda_{v}}+1+\eta_{v}, \quad \eta_{v}>0
$$

gives the strict passivity for the input-output mapping of

$$
\sum_{i=1}^{N}\left(1-u_{i}\right) \tilde{i}_{L, i} \longmapsto \widetilde{v}_{d c}^{*}
$$

In [20], the strict passivity and $\mathscr{L}_{2}$ stability are formally defined, and it is proved that the strict passivity implies that $\mathscr{L}_{2}$ stability. The result of Lemma 1 indicates that the output voltage of $v_{\mathrm{dc}}(t)$ converges to the target output voltage trajectory of $v_{\mathrm{dc}}^{*}(t)$, exponentially, as the inductor current errors of $\widetilde{i}_{L, i}(t)$ vanish, exponentially. For the proof of Lemma 1, a positive definite function is defined as

$$
V_{v}(t):=\frac{C_{0}}{2}\left(\widetilde{v}_{\mathrm{dc}}^{*}(t)\right)^{2}+\frac{1}{2} \widetilde{w}_{v}^{2}(t)+\frac{\gamma_{v}}{2}\left(\widetilde{w}_{v}^{0}(t)\right)^{2},
$$

$$
\forall t \geq 0 \text {. }
$$

Then, the proof can be completed by showing that

$$
\dot{V}_{v}(t) \leq-\alpha_{v} V_{v}(t)+\widetilde{v}_{\mathrm{dc}}^{*}(t) \sum_{i=1}^{N}\left(1-u_{i}(t)\right) \tilde{i}_{L, i}(t)
$$

$\forall t \geq 0$,

for some constant $\alpha_{v}>0$ where $\widetilde{w}_{v}(t):=\widehat{w}_{v}^{0}-\widehat{w}_{v}(t), \forall t \geq$ 0 , and $\widehat{w}_{v}^{0}$ denotes a steady state value of $\widehat{w}_{v}(t), \forall t \geq 0$. See Appendix for details.

For deriving the final control action, write the inductor current-tracking error dynamics using (2) as

$$
\begin{aligned}
L_{0} \dot{\vec{i}}_{L, i}(t)= & L_{0} \dot{i}_{L, i, \mathrm{ref}}(t)-L_{0} \dot{i}_{L, i}(t) \\
= & L_{0} \dot{i}_{L, i, \mathrm{ref}}(t)+\left(1-u_{i}(t)\right) v_{\mathrm{dc}}(t)-v_{\mathrm{in}, 0} \\
& -w_{L, i, o}(t) \\
= & \left(1-u_{i}(t)\right) v_{\mathrm{dc}}(t)-v_{\mathrm{in}, 0}+w_{L, i}(t) \\
= & -v_{\mathrm{dc}}^{*}(t) u_{i}(t)+v_{\mathrm{dc}}^{*}(t)-\left(1-u_{i}(t)\right) \widetilde{v}_{\mathrm{dc}}^{*}(t) \\
& -v_{\mathrm{in}, 0}+w_{L, i}(t), \quad \forall t \geq 0,
\end{aligned}
$$




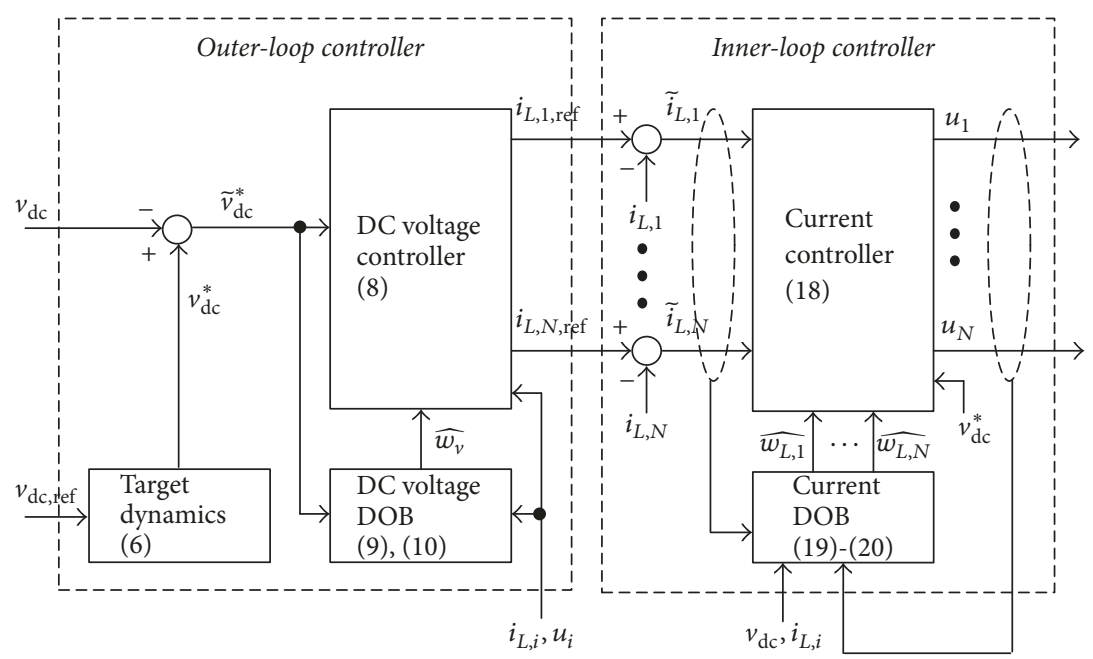

Figure 2: Proposed controller structure.

with $w_{L, i}(t):=L_{0} \dot{i}_{L, i, \text { ref }}(t)-w_{L, i, o}(t), i=1, \ldots, N, \forall t \geq 0$. Then, the stabilization can be accomplished by the proposed stabilizing control law for the duty ratio $u_{i}(t)$ as

$$
\begin{array}{r}
u_{i}(t) \\
=\frac{1}{v_{\mathrm{dc}}^{*}(t)}\left(L_{0} \lambda_{L} \tilde{i}_{L, i}(t)+v_{\mathrm{dc}}^{*}(t)-v_{\mathrm{in}, 0}+\widehat{w}_{L, i}(t)\right), \\
i=1, \ldots, N, \forall t \geq 0,
\end{array}
$$

where the inductor current references $i_{L, i, \text { ref }}(t)$ come from (8), $\lambda_{L}>0$ denotes a tuning parameter, and the estimated disturbances $\widehat{w}_{L, i}(t)$ are given by

$$
\begin{aligned}
\widehat{w}_{L, i}(t):=\zeta_{L, i}(t)+l_{L, i} L_{0} \tilde{i}_{L, i}(t), & \\
& i=1, \ldots, N, \forall t \geq 0,
\end{aligned}
$$

and $\zeta_{L, i}(t)$ stand for the state of the dynamical equations:

$$
\begin{aligned}
\dot{\zeta}_{L, i}(t)= & -l_{L, i} \zeta_{L, i}(t)-l_{L, i}^{2} L_{0} \widetilde{i}_{L, i}(t) \\
& +l_{L, i}\left(-\left(1-u_{i}(t)\right) v_{\mathrm{dc}}(t)+v_{\mathrm{in}, 0}\right), \\
& \quad i=1, \ldots, N, \forall t \geq 0,
\end{aligned}
$$

with $l_{L, i}>0$ being the observer gains. For the rest of this article, the combination of two equations of (20) and (19) is called the inductor current DOB. The proposed controller structure is visualized in Figure 2.

The substitution of the proposed control law of (18) to the inductor current error dynamics of (17) yields that

$$
\begin{aligned}
L_{0} \dot{\vec{i}}_{L, i}(t)= & -L_{0} \lambda_{L} \tilde{i}_{L, i}(t)+\Delta w_{L, i}(t) \\
& -\left(1-u_{i}(t)\right) \widetilde{v}_{\mathrm{dc}}^{*}(t), \\
& \quad i=1, \ldots, N, \forall t \geq 0,
\end{aligned}
$$

where $\Delta w_{L, i}(t):=w_{L, i}(t)-\widehat{w}_{L, i}(t), i=1, \ldots, N, \forall t \geq 0$. Lemma 2 gives the closed-loop property of (21), which is used for analyzing the closed-loop stability.
Lemma 2. Assuming that the disturbance of $w_{L, i}(t)$ converges to its steady state value $w_{L, i}^{0}$, exponentially, that is, there are constants of $k_{L, i}>0$ such that

$$
\dot{\vec{w}}_{L, i}^{0}(t)=-k_{L, i} \widetilde{w}_{L, i}^{0}(t), \quad i=1, \ldots, N, \forall t>0,
$$

where $\widetilde{w}_{L, i}^{0}(t):=w_{L, i}^{0}-w_{L, i}(t), \forall t \geq 0$, then, a specified design parameter of $\lambda_{L}>0$ with the DOB gains of

$$
l_{L, i}=\frac{3}{4 L_{0} \lambda_{L}}+1+\eta_{L, i}, \quad \eta_{L, i}>0, i=1, \ldots, N
$$

gives the strict passivity for the input-output mapping of

$$
-\left(1-u_{i}(t)\right) \widetilde{v}_{d c}^{*}(t) \longmapsto \widetilde{i}_{L, i}(t), \quad i=1, \ldots, N .
$$

Lemma 2 shows that the inductor current-tracking error of $\tilde{i}_{L, i}(t)$ vanishes exponentially if the output voltage-tracking error of $\widetilde{v}_{\mathrm{dc}}^{*}(t)$ does so. For the proof, a positive definite function is defined as

$$
\begin{aligned}
V_{L}(t):= & \frac{L_{0}}{2} \sum_{i=1}^{N} \widetilde{i}_{L, i}^{2}(t)+\sum_{i=1}^{N} \frac{1}{2} \widetilde{w}_{L, i}^{2}(t) \\
& +\sum_{i=1}^{N} \frac{\gamma_{L, i}}{2}\left(\widetilde{w}_{L, i}^{0}(t)\right)^{2}, \quad \forall t \geq 0 .
\end{aligned}
$$

The proof can be completed by showing that

$$
\dot{V}_{L}(t) \leq-\alpha_{L} V_{L}(t)-\sum_{i=1}^{N} \tilde{i}_{L, i}\left(1-u_{i}(t)\right) \widetilde{v}_{\mathrm{dc}}^{*}(t),
$$

$\forall t \geq 0$

for some constant $\alpha_{L}>0$ where

$$
\widetilde{w}_{L}(t):=\widehat{w}_{L}^{0}-\widehat{w}_{L}(t), \quad \forall t \geq 0,
$$

where $\widehat{w}_{L}^{0}$ denotes a steady state value of $\widehat{w}_{L}(t), \forall t \geq 0$. See Appendix for details. 
Finally, Theorem 3 asserts that the proposed control algorithm forces the output voltage trajectory to be exponentially convergent to the target output voltage trajectory coming from the LPF dynamics of (6). The two resulting inequalities of (16) and (26) given by Lemmas 1 and 2 play the crucial role of proving Theorem 3, and the proof is given in Appendix.

Theorem 3. Assuming that the assumptions of Lemmas 1 and 2 hold true, then, the proposed control law of (8), (18) with the DOBs of (9), (10), (19), and (20) forces the closed-loop system to recover the target output voltage-tracking performance of (6), exponentially; that is,

$$
\left|\tilde{v}_{d c}^{*}(t)\right| \leq \kappa e^{-(\alpha / 2) t}, \quad \forall t \geq 0,
$$

for some positive constants $\kappa_{c l}$ and $\alpha_{c l}$.

Note that, interestingly, although the proposed proportional-type control law of (8) and (18) with the DOBs of (9), (10), (19), and (20) does not include the tracking error integrators, it ensures the offset-free property, which is a practical advantage of this article. See Theorem 4 for details, and the proof is included in Appendix.

Theorem 4. The closed-loop system always eliminates the offset errors of the output voltage in the steady state. That is,

$$
v_{d c}^{0}=v_{d c, r e f}^{0},
$$

where $v_{d c}^{0}$ and $v_{d c \text {,ref }}^{0}$ represent the steady-state of $v_{d c}(t)$ and $v_{d c, \text { ref }}(t)$, respectively.

Remark 5. The current and output voltage DOB dynamics can be described in a first-order LPF form (for details, see Appendix):

$$
\begin{aligned}
\dot{\widehat{w}}_{v}(t) & =l_{v}\left(w_{v}(t)-\widehat{w}_{v}(t)\right), \\
\dot{\widehat{w}}_{L, i}(t) & =l_{L, i}\left(w_{L, i}(t)-\widehat{w}_{L, i}(t)\right), \\
& i=1, \ldots, N, \forall t \geq 0 .
\end{aligned}
$$

The corresponding transfer functions are given by

$$
\begin{aligned}
\frac{\widehat{W}_{v}(s)}{W_{v}(s)} & =\frac{l_{v}}{s+l_{v}}, \\
\frac{\widehat{W}_{L, i}(s)}{W_{L, i}(s)} & =\frac{l_{L, i}}{s+l_{L, i}}, \\
& i=1, \ldots, N, \forall s \in \mathbb{C},
\end{aligned}
$$

which implies that the DOB gains of $l_{v}, l_{L, i}>0, i=1, \ldots, N$, can be adjusted for a cut-off frequency ( $\mathrm{rad} / \mathrm{s})$ of the transfer functions of (31).

\section{Simulations}

This section evaluates the output voltage-tracking performance between the proposed and FL methods using the
PowerSIM (PSIM) software with the DLL block embedding the control algorithms in the C-language. To this end, a fourphase interleaved DC/DC boost converter was considered with the parameters

$$
\begin{aligned}
& L=40 \mu \mathrm{H}, \\
& C=1650 \mu \mathrm{F} .
\end{aligned}
$$

The input DC source voltage was set to be $v_{\text {in }}=50 \mathrm{~V}$, and pulse-width modulation (PWM) and the control frequencies were both chosen as $20 \mathrm{kHz}$. In order to take the parameter uncertainties into account, the nominal parameters of the converter were assumed to be

$$
\begin{aligned}
& L_{0}=28 \mu \mathrm{H}(=0.7 L), \\
& C_{0}=2145 \mu \mathrm{F}(=1.3 C),
\end{aligned}
$$

which are adopted to constitute the control algorithms. Figure 3 visualizes the implementation of the closed-loop system.

The FL controller in [7] was used for a comparison given as

$$
\begin{aligned}
& u_{i}(t)=\frac{1}{v_{\mathrm{dc}}(t)}\left(-R_{\mathrm{dc}} i_{L, i}(t)+L_{0} \omega_{\mathrm{cc}} \widetilde{i}_{L, i}(t)\right. \\
& \left.+R_{\mathrm{dc}} \omega_{\mathrm{cc}} \int_{0}^{t} \widetilde{i}_{L, i}(\tau) d \tau+\left(v_{\mathrm{dc}}(t)-v_{\mathrm{in}}\right)\right), \\
& i_{L, i, \mathrm{ref}}(t)=\frac{1}{4}\left(-R_{\mathrm{dv}} v_{\mathrm{dc}}(t)+C_{0} \omega_{\mathrm{vc}} \widetilde{v}_{\mathrm{dc}}(t)\right. \\
& \left.+R_{\mathrm{dv}} \omega_{\mathrm{vc}} \int_{0}^{t} \widetilde{v}_{\mathrm{dc}}(\tau) d \tau\right), \quad i=1, \ldots, 4, \forall t \geq 0,
\end{aligned}
$$

which yields the closed-loop transfer functions of the inner and outer-loops through the pole-zero cancellations: $V_{\mathrm{dc}}(s) / V_{\mathrm{dc}, \mathrm{ref}}(s)=\omega_{\mathrm{vc}} /\left(s+\omega_{\mathrm{vc}}\right), I_{L, i}(s) / I_{L, i, \mathrm{ref}}(s)=\omega_{\mathrm{cc}} /\left(s+\omega_{\mathrm{cc}}\right)$, $i=1, \ldots, 4, \forall s \in \mathbb{C}$ with the adjustable active damping coefficients $R_{\mathrm{dc}}>0, R_{\mathrm{dv}}>0$, and the cut-off frequencies $\omega_{\mathrm{vc}}>0, \omega_{\mathrm{cc}}>0$. The corresponding $f_{\mathrm{vc}}, f_{\mathrm{cc}}$ were tuned to be $f_{\mathrm{vc}}=15 \mathrm{~Hz}, f_{\mathrm{cc}}=1000 \mathrm{~Hz}$ so that $\omega_{\mathrm{vc}}=2 \pi f_{\mathrm{vc}}=94.2 \mathrm{rad} / \mathrm{s}$, $\omega_{\mathrm{cc}}=2 \pi f_{\mathrm{cc}}=6280 \mathrm{rad} / \mathrm{s}$, and the active damping coefficients were selected as $R_{\mathrm{dc}}=R_{\mathrm{dv}}=0.1$. Note that the output voltage error decay ratio parameter $\lambda_{v}$ of the proposed method was set to be the same as $\omega_{\mathrm{vc}}$, and the design parameter $\lambda_{L}$ was also let to be the same as the cut-off frequency of $\omega_{\text {cc }}$ for a fair comparison because the inductor current error dynamics of (21) can be approximately given as a first-order LPF form: $\dot{i}_{L, i}(t)=-\lambda_{L}\left(i_{L, i}(t)-i_{L, i, \text { ref }}(t)\right), i=1, \ldots, N, \forall t \geq 0$, for a slowly time varying inductor current reference signal of $i_{L, i, \text { ref }}(t)$. The DOB gains were tuned as $l_{v}=l_{L, i}=1256 \mathrm{rad} / \mathrm{s}$, $i=1, \ldots, N$, for the cut-off frequency of the corresponding transfer functions in (31) to be $200 \mathrm{~Hz}$, which also meets the assumptions of (13)-(23).

In the first stage, the evaluation of the output voltagetracking performance with the resistive load of $R_{L}=20 \Omega$ was performed where the output voltage reference was increased from $100 \mathrm{~V}$ to $150 \mathrm{~V}$, and it was decreased to $120 \mathrm{~V}$. From 


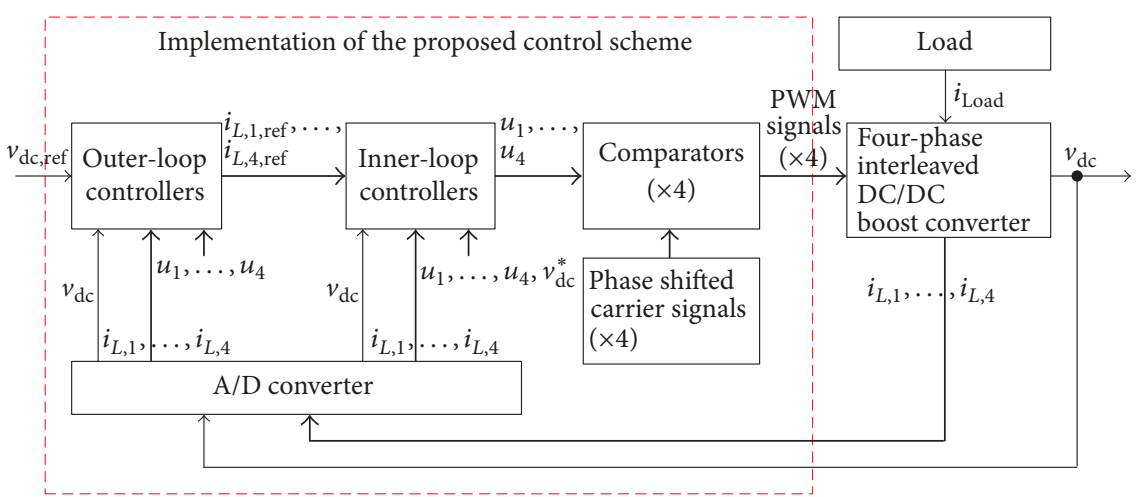

Figure 3: Implementation of the closed-loop system.
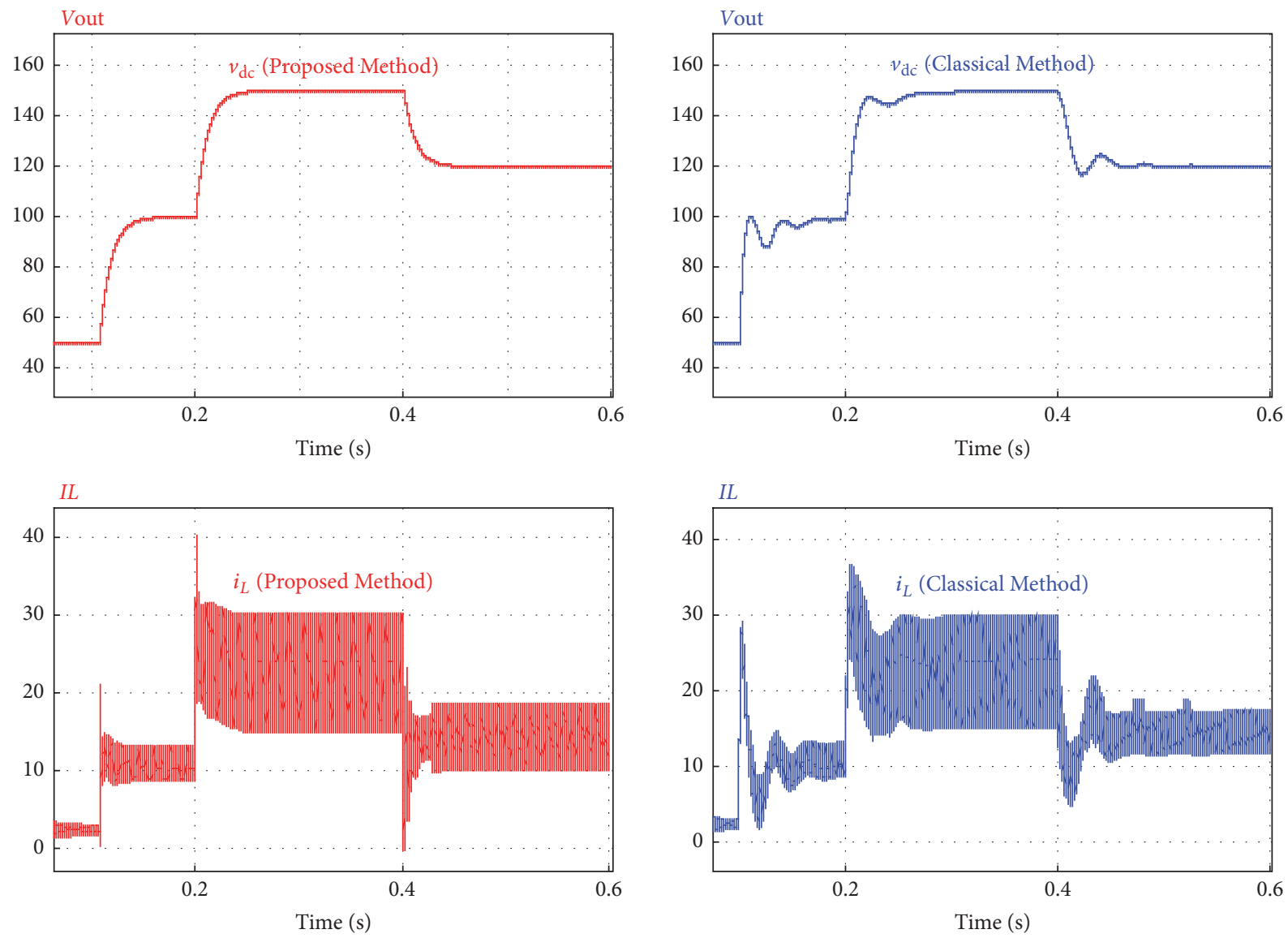

FIGURE 4: Output voltage-tracking performance comparison between proposed and FL methods with resistive load of $R_{L}=20 \Omega$.

the simulation results depicted in Figure 4, the proposed method successfully maintains the closed-loop performance to be the desired first-order LPF characteristic despite the parameter mismatches. Figure 5 presents the state behaviors of the DOBs.

In the second stage, the evaluation of the closed-loop robustness was carried out through investigating the output voltage-tracking performance variations under the same output voltage reference with the different resistive loads, $R_{L}=7.5,20,30 \Omega$. As can be seen from the results given in Figure 6, the proposed method effectively reduces the voltage-tracking performance changes for several different loads. This is a sharp contrast to the FL method.

In this simulation setting, the closed-loop tracking performance was quantitatively compared for the resistive loads, $R_{L}=50,30,20,10 \Omega$, using the two cost functions defined as

$$
\begin{aligned}
J_{\text {int }} & :=\int_{0}^{\infty}\left|v_{\mathrm{dc}}^{*}(t)-v_{\mathrm{dc}}(t)\right| d t, \\
J_{\max } & :=\max \left|v_{\mathrm{dc}}^{*}(t)-v_{\mathrm{dc}}(t)\right|, \quad \forall t \geq 0,
\end{aligned}
$$


TABLE 1: Quantitative output voltage-tracking performance comparison for resistive loads, $R_{L}=50,30,20,10 \Omega$.

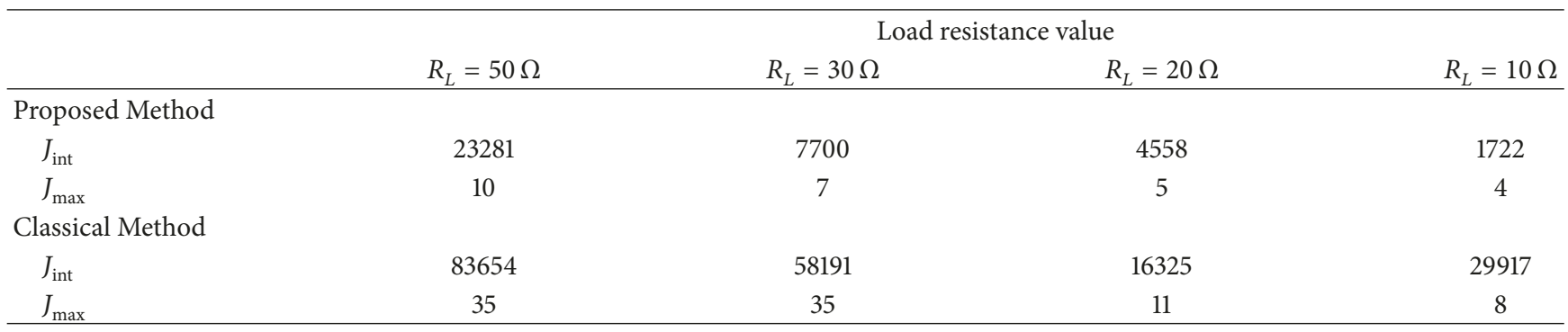

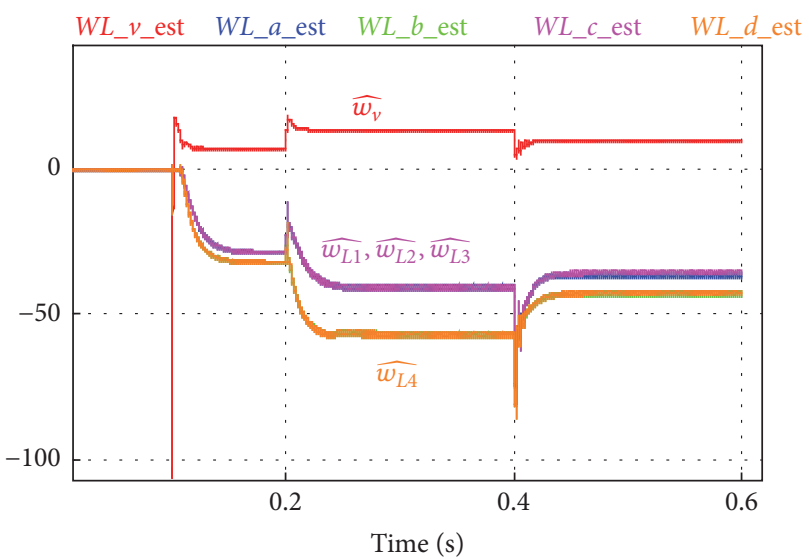

FIgURE 5: Disturbance estimation behaviors.

which implies that the best controller would guarantee the two cost functions of (35) to be minimized for all operating points. The resulting table is given in Table 1 , which shows that the proposed method offers a better tracking performance than the FL method at least two times for these four operating points.

In the last stage, the evaluation of the output voltage regulation performance was conducted by using the pulse resistive load from $R_{L}=15 \Omega$ to $R_{L}=7.5 \Omega$ at the $150 \mathrm{~V}$ operation mode. Figure 7 depicts the output voltage regulation comparison results with the corresponding inductor current, which implies that the proposed method effectively reduces the over/under shoots caused by the load variation, comparing the FL method.

\section{Experimental Results}

This section experimentally verifies the performance of the proposed method by comparing it with the FL technique used in the previous section. In this experiment, a $3-\mathrm{kW}$ four-phase interleaved DC/DC converter shown in Figure 1 was utilized with the input DC source voltage level of $v_{\text {in }}=$ $50 \mathrm{~V}$, and the digital signal processor (DSP) of TMS320F28335 was used for implementing the proposed and FL control algorithms with the control and PWM periods of $T_{s}=50 \mu \mathrm{s}$. The experimental setup is shown in Figure 8.

Except for design parameters of $\omega_{\mathrm{vc}}, \omega_{\mathrm{cc}}, \lambda_{v}$, and $\lambda_{L}$, the control parameters of two controllers were left the same as that in the simulation section. These four parameters
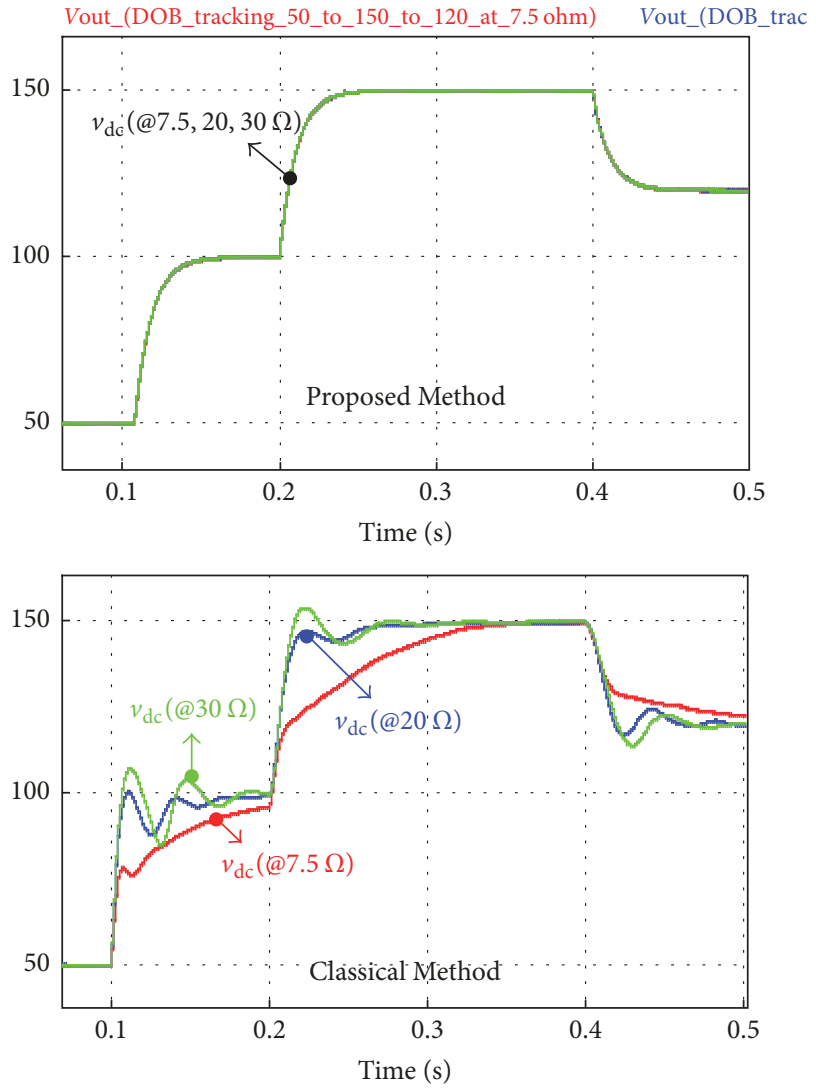

FIGURE 6: Output voltage-tracking performance comparison between proposed and FL methods at three resistive loads, $R_{L}=7.5,20,30 \Omega$.

were selected as $\omega_{\mathrm{vc}}=31.4 \mathrm{rad} / \mathrm{s}\left(f_{\mathrm{vc}}=5 \mathrm{~Hz}\right), \omega_{\mathrm{cc}}=$ $2512 \mathrm{rad} / \mathrm{s}\left(f_{\mathrm{cc}}=400 \mathrm{~Hz}\right)$, and $\lambda_{v}=\omega_{\mathrm{vc}}, \lambda_{L}=\omega_{\mathrm{cc}}$.

In the first stage, the evaluation of the output voltagetracking performance was experimentally performed with an increasing output voltage reference from $75 \mathrm{~V}$ to $100 \mathrm{~V}$ under the resistive loads of $R_{L}=6.6,20 \Omega$. From Figures 9 and 10, as, intended, the proposed controller successfully forces the output voltage dynamics to be the desired trajectory coming from (6) for the different operation modes.

The second stage examines the output voltage regulation performance regarding two step load changes; the resistive load was changed from $20 \Omega$ to $10 \Omega$ and from $10 \Omega$ to $6.6 \Omega$, in a sequential manner. The resulting waveforms are shown 

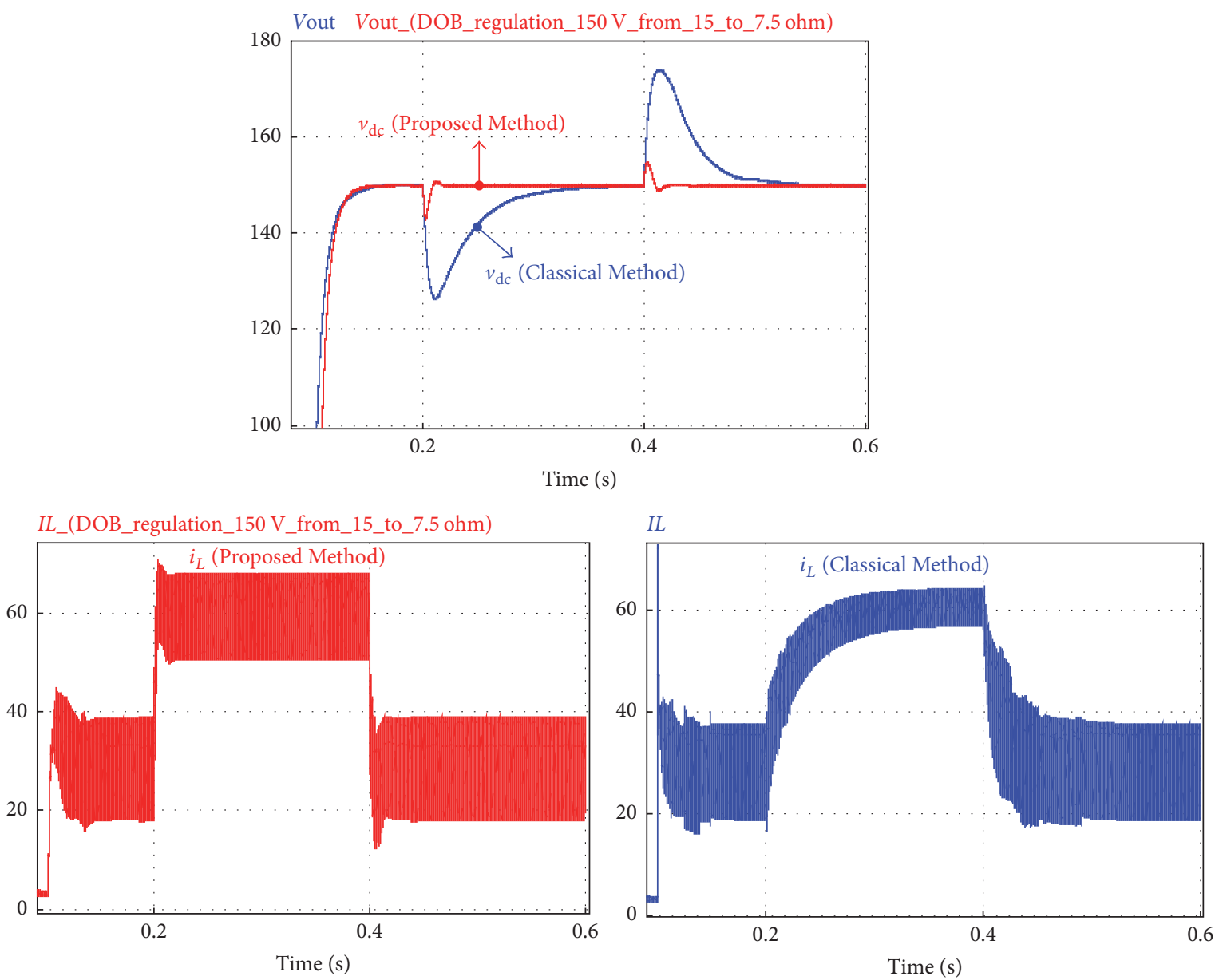

FIGURE 7: Output voltage regulation performance comparison between proposed and FL methods at the output voltage $150 \mathrm{~V}$ with the pulse resistive load from $R_{L}=15 \Omega$ to $R_{L}=7.5 \Omega$.

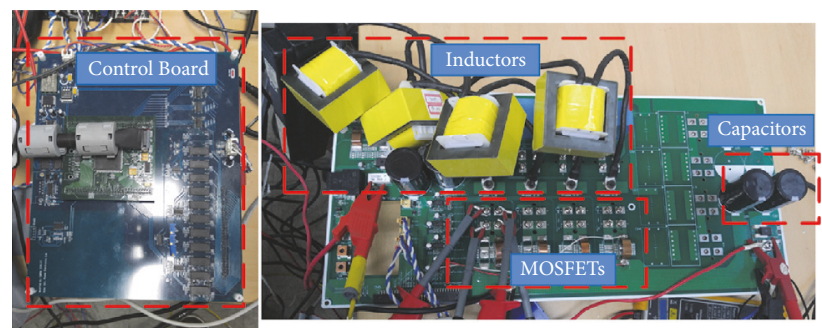

Figure 8: Experimental setup.

in Figures 11 and 12, which demonstrates that the proposed method effectively enhances the output voltage regulation performance through a reduction of the undershoot originated from the abrupt load variation.

From these simulation and experimental results, it can be concluded that the proposed method has the two practical merits:

(1) The proposed method would suggest an almost same closed-loop performance for various operating ranges without any gain scheduling method.
(2) The proposed method simplifies the control algorithms by getting rid of the use of the tracking error integrators with the antiwindup algorithms.

\section{Conclusions}

This paper suggests a performance recovery output voltagetracking controller for an unknown $N$-phase interleaved DC/DC boost converter. Taking the parameter and load current uncertainties into account, the proposed control law was devised for the output voltage dynamics to converge to the desired behavior coming from a LPF despite the parameter and load uncertainties. Various simulation and experimental evidences confirmed that the proposed technique would provide a better closed-loop performance for several industrial applications.

\section{Appendix}

This section provides the proofs of lemmas and theorems. The proof of Lemma 1 is given as follows. 

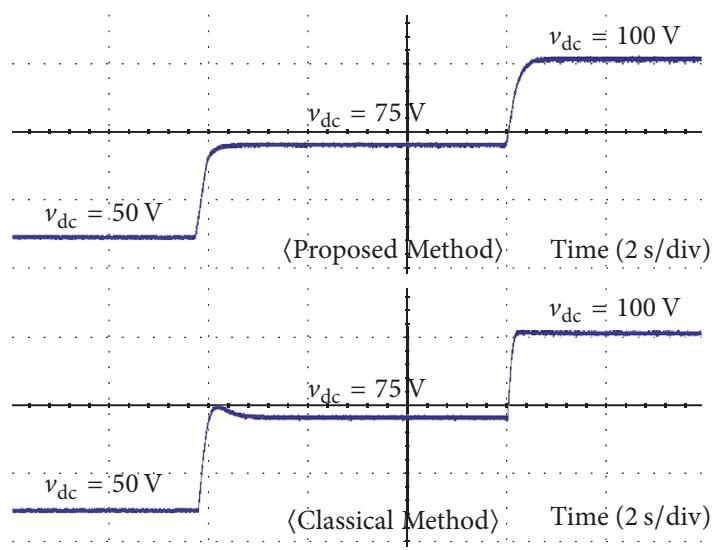

$-v_{\mathrm{dc}}(20 \mathrm{~V} / \mathrm{div})$

FIGURE 9: Experimental results of output voltage-tracking performance comparison between proposed method and FL methods with resistive load $R_{L}=6.6 \Omega$.
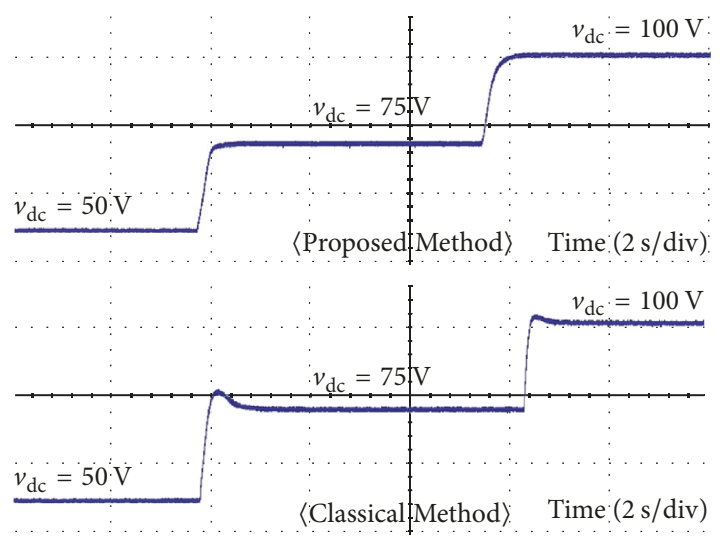

$-v_{\mathrm{dc}}(20 \mathrm{~V} / \mathrm{div})$

FIGURE 10: Experimental results of output voltage-tracking performance comparison between proposed and FL methods with resistive load $R_{L}=20 \Omega$.

Proof. The combination of the nonlinear observer of (10) and the output of (9) leads to

$$
\begin{aligned}
\dot{\widehat{\omega}}_{v}-l_{v} C_{0} \dot{\tilde{v}}_{\mathrm{dc}}^{*}= & -l_{v}\left(\widehat{w}_{v}-l_{v} C_{0} \widetilde{v}_{\mathrm{dc}}^{*}\right)-l_{v}^{2} C_{0} \widetilde{v}_{\mathrm{dc}}^{*} \\
& +l_{v} \sum_{i=1}^{N}\left(1-u_{i}\right) i_{L, i}, \quad \forall t \geq 0,
\end{aligned}
$$

which gives that (see the output voltage error dynamics of (7))

$$
\begin{aligned}
\dot{\hat{w}}_{v} & =l_{v}\left(C_{0} \dot{\tilde{\vec{v}}}_{\mathrm{dc}}^{*}+\sum_{i=1}^{N}\left(1-u_{i}\right) i_{L, i}-\widehat{w}_{v}\right) \\
& =l_{v}\left(w_{v}-\widehat{w}_{v}\right), \quad \forall t \geq 0 .
\end{aligned}
$$
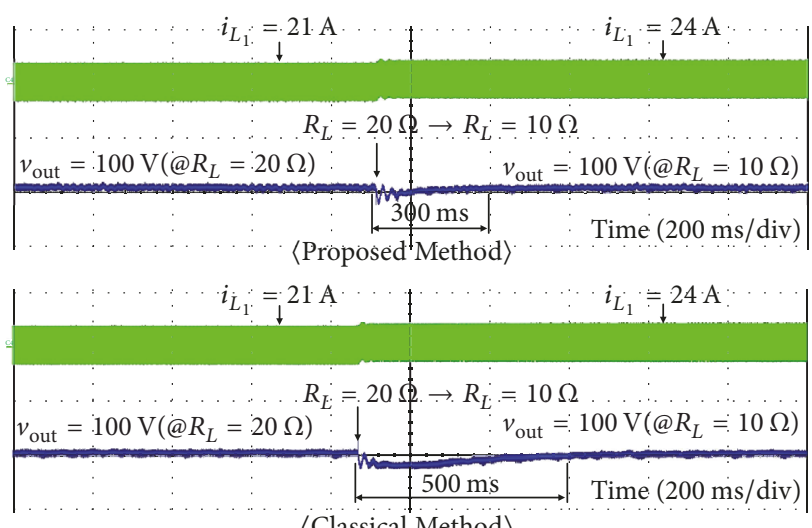

$$
\begin{aligned}
& -i_{L_{1}}(50 \mathrm{~A} / \mathrm{div}) \\
& -v_{\text {out }}(10 \mathrm{~V} / \mathrm{div})
\end{aligned}
$$

FIGURE 11: Experimental results of output voltage regulation performance comparison between proposed and FL methods regarding to resistive load variation from $R_{L}=20 \Omega$ to $R_{L}=10 \Omega$.
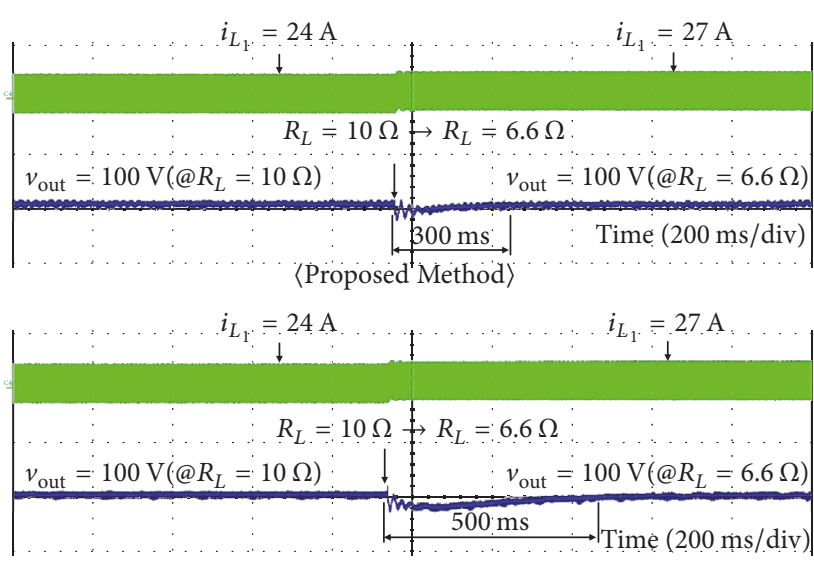

$\langle$ Classical Method

$i_{L_{1}}(50 \mathrm{~A} / \mathrm{div})$

- $v_{\text {out }}(10 \mathrm{~V} /$ div $)$

FIGURE 12: Experimental results of output voltage regulation performance comparison between proposed and FL methods regarding to resistive load variation from $R_{L}=10 \Omega$ to $R_{L}=6.6 \Omega$.

The assumption of (12) results in the steady state DOB dynamics as (see (A.2))

$$
0=l_{v}\left(w_{v}^{0}-\widehat{w}_{v}^{0}\right)
$$

which means that

$$
w_{v}^{0}=\widehat{w}_{v}^{0}
$$

Equation (A.4) implies that

$$
\begin{aligned}
\dot{\widetilde{w}}_{v} & =-l_{v}\left(\widetilde{w}_{v}-\widetilde{w}_{v}^{0}\right), \\
\Delta w_{v} & =\widetilde{w}_{v}-\widetilde{w}_{v}^{0}, \quad \forall t \geq 0,
\end{aligned}
$$


where $\widetilde{w}_{v}:=\widehat{w}_{v}^{0}-\widehat{w}_{v}, \forall t \geq 0$. Together with (A.6), it is possible to rewrite the tracking error dynamics of (11) as

$$
C_{0} \dot{\tilde{v}}_{\mathrm{dc}}^{*}=-C_{0} \lambda_{v} \widetilde{v}_{\mathrm{dc}}^{*}+\widetilde{w}_{v}-\widetilde{w}_{v}^{0}+\sum_{i=1}^{N}\left(1-u_{i}\right) \tilde{i}_{L, i}
$$

$\forall t \geq 0$.

Now consider the positive definite function of (15)

$$
V_{v}=\frac{C_{0}}{2}\left(\widetilde{v}_{\mathrm{dc}}^{*}\right)^{2}+\frac{1}{2} \widetilde{w}_{v}^{2}+\frac{\gamma_{v}}{2}\left(\widetilde{w}_{v}^{0}\right)^{2}, \quad \forall t \geq 0,
$$

with $\gamma_{v}$ denoting positive constant to be found later. Then, the time-derivative of $\dot{V}_{v}$ can be obtained by using the closed-loop trajectories of (12), (A.5), and (A.7):

$$
\begin{aligned}
\dot{V}_{v}= & \widetilde{v}_{\mathrm{dc}}^{*}\left(-C_{0} \lambda_{v} \widetilde{v}_{\mathrm{dc}}^{*}+\widetilde{w}_{v}-\widetilde{w}_{v}^{0}+\sum_{i=1}^{N}\left(1-u_{i}\right) \widetilde{i}_{L, i}\right) \\
& -l_{v} \widetilde{w}_{v}^{2}+l_{v} \widetilde{w}_{v} \widetilde{w}_{v}^{0}-\gamma_{v} k_{v}\left(\widetilde{w}_{v}^{0}\right)^{2} \\
\leq & -\frac{C_{0} \lambda_{v}}{3}\left(\widetilde{v}_{\mathrm{dc}}^{*}\right)^{2}-\left(l_{v}-\frac{3}{4 C_{0} \lambda_{v}}-1\right) \widetilde{w}_{v}^{2} \\
& -\left(\gamma_{v} k_{v}-\frac{3}{4 C_{0} \lambda_{v}}-\frac{l_{v}^{2}}{4}\right)\left(\widetilde{w}_{v}^{0}\right)^{2} \\
& +\widetilde{v}_{\mathrm{dc}}^{*} \sum_{i=1}^{N}\left(1-u_{i}\right) \widetilde{i}_{L, i}, \quad \forall t \geq 0,
\end{aligned}
$$

where the inequality is confirmed by Young's inequality:

$$
x y \leq \frac{\epsilon}{2} x^{2}+\frac{1}{2 \epsilon} y^{2}, \quad \forall x, y \in \mathbb{R}, \forall \epsilon>0 .
$$

It is easy to verify that the DOB gains of (13) with the positive constant of $\gamma_{v}$ given as $\gamma_{v}=\left(1 / k_{v}\right)\left(3 / 4 C_{0} \lambda_{v}+l_{v}^{2} / 4+1 / 2\right)$ renders the inequality of (A.9) as

$$
\begin{aligned}
\dot{V}_{v} \leq & -\frac{C_{0} \lambda_{v}}{3}\left(\widetilde{v}_{\mathrm{dc}}^{*}\right)^{2}-\eta_{v} \widetilde{w}_{v}^{2}-\frac{1}{2}\left(\widetilde{w}_{v}^{0}\right)^{2} \\
& +\widetilde{v}_{\mathrm{dc}}^{*} \sum_{i=1}^{N}\left(1-u_{i}\right) \widetilde{i}_{L, i} \\
\leq & -\alpha_{v} V_{v}+\widetilde{v}_{\mathrm{dc}}^{*} \sum_{i=1}^{N}\left(1-u_{i}\right) \widetilde{i}_{L, i}, \quad \forall t \geq 0,
\end{aligned}
$$

with $\alpha_{v}:=\min \left\{2 \lambda_{v} / 3,2 \eta_{v}, 1 / \gamma_{v}\right\}$, which confirms the strict passivity regarding to the input-output mapping of (14).

The proof of Lemma 2 is given as follows.

Proof. The combination of the nonlinear observer of (20) and the output of (19) leads to

$$
\begin{aligned}
\dot{\hat{w}}_{L, i}-l_{L, i} L_{0} \dot{\vec{i}}_{L, i}= & -l_{L, i}\left(\widehat{w}_{L, i}-l_{L, i} L_{0} \tilde{i}_{L, i}\right)-l_{L, i}^{2} L_{0} \tilde{i}_{L, i} \\
& +l_{L, i}\left(-\left(1-u_{i}\right) v_{\mathrm{dc}}+v_{\mathrm{in}, 0}\right),
\end{aligned}
$$

$\forall t \geq 0$, which gives that (see the output voltage error dynamics of (17)):

$$
\begin{aligned}
\dot{\widehat{\vec{w}}}_{L, i} & =l_{L, i}\left(L_{0} \dot{\overrightarrow{\dot{i}}}_{L, i}-\left(1-u_{i}\right) v_{\mathrm{dc}}+v_{\mathrm{in}, 0}-\widehat{w}_{L, i}\right) \\
& =l_{L, i}\left(w_{L, i}-\widehat{w}_{L, i}\right), \quad i=1, \ldots, N, \quad \forall t \geq 0 .
\end{aligned}
$$

The assumption of (22) results in the steady state DOB dynamics as $(\operatorname{see}(\mathrm{A} .13)) 0=l_{L, i}\left(w_{L, i}^{0}-\widehat{w}_{L, i}^{0}\right), i=1, \ldots, N$, which means that

$$
w_{L, i}^{0}=\widehat{w}_{L, i}^{0}, \quad i=1, \ldots, N .
$$

The equation of (A.14) implies that:

$$
\begin{aligned}
\dot{\tilde{w}}_{L, i} & =-l_{L, i}\left(\widetilde{w}_{L, i}-\widetilde{w}_{L, i}^{0}\right), \\
\Delta w_{L, i} & =\widetilde{w}_{L, i}-\widetilde{w}_{L, i}^{0}, \quad i=1, \ldots, N, \quad \forall t \geq 0,
\end{aligned}
$$

where $\widetilde{w}_{L, i}:=\widehat{w}_{L, i}^{0}-\widehat{w}_{L, i}, \forall t \geq 0$. Together with (A.16), it is possible to rewrite the error dynamics of (21) as

$$
\begin{aligned}
& L_{0} \dot{\vec{i}}_{L, i}=-L_{0} \lambda_{L} \widetilde{i}_{L, i}+\widetilde{w}_{L, i}-\widetilde{w}_{L, i}^{0}-\left(1-u_{i}\right) \widetilde{v}_{\mathrm{dc}}^{*}, \\
& i=1, \ldots, N, \forall t \geq 0 .
\end{aligned}
$$

Now consider the positive definite function in (25):

$$
V_{L}=\frac{L_{0}}{2} \sum_{i=1}^{N} \widetilde{i}_{L, i}^{2}+\sum_{i=1}^{N} \frac{1}{2} \widetilde{w}_{L, i}^{2}+\sum_{i=1}^{N} \frac{\gamma_{L, i}}{2}\left(\widetilde{w}_{L, i}^{0}\right)^{2}
$$

$\forall t \geq 0$,

with $\gamma_{L, i}, i=1, \ldots, N$, being positive constants to be found later. The time-derivative of $\dot{V}_{L}$ can be obtained by using the closed-loop trajectories of (22), (A.15), and (A.17):

$$
\begin{aligned}
\dot{V}_{L}= & \sum_{i=1}^{N} \widetilde{i}_{L, i}\left(-L_{0} \lambda_{L} \widetilde{i}_{L, i}+\widetilde{w}_{L, i}-\widetilde{w}_{L, i}^{0}-\left(1-u_{i}\right) \widetilde{v}_{\mathrm{dc}}^{*}\right) \\
& -\sum_{i=1}^{N} l_{L, i} \widetilde{w}_{L, i}^{2}+\sum_{i=1}^{N} l_{L, i} \widetilde{w}_{L, i} \widetilde{w}_{L, i}^{0} \\
& -\sum_{i=1}^{N} \gamma_{L, i} k_{L, i}\left(\widetilde{w}_{L, i}^{0}\right)^{2} \\
\leq & -\frac{L_{0} \lambda_{L} \sum_{i=1}^{N} \widetilde{i}_{L, i}^{2}-\sum_{i=1}^{N}\left(l_{L, i}-\frac{3}{4 L_{0} \lambda_{L}}-1\right) \widetilde{w}_{L, i}^{2}}{3} \quad-\sum_{i=1}^{N}\left(\gamma_{L, i} k_{L, i}-\frac{3}{4 L_{0} \lambda_{L}}-\frac{l_{L, i}^{2}}{4}\right)\left(\widetilde{w}_{L, i}^{0}\right)^{2} \\
& -\sum_{i=1}^{N} \tilde{i}_{L, i}\left(1-u_{i}\right) \widetilde{v}_{\mathrm{dc}}^{*} \quad \forall t \geq 0,
\end{aligned}
$$

where the inequality is confirmed by Young's inequality of (A.10). It is easy to verify that the DOB gains of (23) with the 
positive constants of $\gamma_{L, i}$, given as $\gamma_{L, i}=\left(1 / k_{L, i}\right)\left(3 / 4 L_{0} \lambda_{L}+\right.$ $\left.l_{L, i}^{2} / 4+1 / 2\right), i=1, \ldots, N$, render the inequality of (A.19) as

$$
\begin{aligned}
\dot{V}_{L} \leq & -\frac{L_{0} \lambda_{L}}{3} \sum_{i=1}^{N} \widetilde{i}_{L, i}^{2}-\sum_{i=1}^{N} \eta_{L, i} \widetilde{w}_{L, i}^{2}-\sum_{i=1}^{N} \frac{1}{2}\left(\widetilde{w}_{L, i}^{0}\right)^{2} \\
& -\sum_{i=1}^{N} \widetilde{i}_{L, i}\left(1-u_{i}\right) \widetilde{v}_{\mathrm{dc}}^{*} \\
\leq & -\alpha_{L} V-\sum_{i=1}^{N} \widetilde{i}_{L, i}\left(1-u_{i}\right) \widetilde{v}_{\mathrm{dc}}^{*}, \quad \forall t \geq 0,
\end{aligned}
$$

with $\alpha_{L}:=\min \left\{2 \lambda_{L} / 3,2 \eta_{L, 1}, \ldots, 2 \eta_{L, N}, 1 / \gamma_{L, i}, \ldots, 1 / \gamma_{L, N}\right\}$ which confirms the strict passivity of $(24)$.

The proof of Theorem 3 is given as follows.

Proof. The composite-type positive definite function defined as

$$
V_{\mathrm{cl}}:=V_{v}+V_{L}, \quad \forall t \geq 0,
$$

with two positive defined functions given in (15) and (25), gives its time-derivative of $\dot{V}_{\mathrm{cl}}$ using the closed-loop trajectories of (A.7) and (A.17) as

$$
\begin{aligned}
\dot{V}_{\mathrm{cl}}= & \dot{V}_{v}+\dot{V}_{L} \\
\leq & -\alpha_{v} V_{v}+\widetilde{v}_{\mathrm{dc}}^{*} \sum_{i=1}^{N}\left(1-u_{i}\right) \widetilde{i}_{L, i}-\alpha_{L} V \\
& -\sum_{i=1}^{N} \widetilde{i}_{L, i}\left(1-u_{i}\right) \widetilde{v}_{\mathrm{dc}}^{*} \leq-\alpha_{\mathrm{cl}} V_{\mathrm{cl}}, \quad \forall t \geq 0,
\end{aligned}
$$

where the two inequalities of (16) and (26) give the first inequality, and the positive constant $\alpha_{\mathrm{cl}}$ is defined as $\alpha_{\mathrm{cl}}:=$ $\min \left\{\alpha_{v}, \alpha_{L}\right\}$ which confirms the inequality of (28) by the Comparison principle in [20].

The proof of Theorem 4 is given as follows.

Proof. As can be seen in the proof of Theorem 3 in Appendix, the inductor current and output voltage DOB dynamics can be equivalently written as $\dot{\widehat{w}}_{v}=l_{v} \Delta w_{v}, \dot{\widehat{w}}_{L, i}=l_{L, i} \Delta w_{L, i}, i=$ $1, \ldots, N, \forall t \geq 0$, which yields the steady-state equations

$$
\begin{aligned}
& \Delta w_{v}^{0}=0, \\
& \Delta w_{L, i}^{0}=0, \\
& \forall l_{v}, l_{L, i}>0, \quad i=1, \ldots, N,
\end{aligned}
$$

where $\Delta w_{v}^{0}, \Delta w_{L, i}^{0}$ denote to the steady-states of $\Delta w_{v}(t)$, $\Delta w_{L, i}(t), i=1, \ldots, N$, respectively. The combination of the closed-loop error dynamics of (11) and (21) and the target output voltage dynamics of (6) gives the steady-state equations

$$
\begin{aligned}
& \mathbf{0}=(\mathbf{J}-\mathbf{R}) \widetilde{\mathbf{x}}, \\
& 0=-\omega_{\mathrm{vc}}\left(\left(v_{\mathrm{dc}}^{*}\right)^{0}-v_{\mathrm{dc}, \mathrm{ref}}^{0}\right),
\end{aligned}
$$

where

$$
\begin{aligned}
& \mathbf{J}=\left[\begin{array}{cccc}
0 & \left(1-u_{1}^{0}\right) & \cdots & \left(1-u_{N}^{0}\right) \\
-\left(1-u_{1}^{0}\right) & 0 & \cdots & 0 \\
\vdots & \vdots & \ddots & \vdots \\
-\left(1-u_{N}^{0}\right) & 0 & \cdots & 0
\end{array}\right] \\
& \mathbf{R}=\operatorname{diag}\left\{C_{0} \lambda_{v}, L_{0} \lambda_{L}, \ldots, L_{0} \lambda_{L}\right\}, \\
& \widetilde{\mathbf{x}}=\left[\begin{array}{llll}
\left(\widetilde{v}_{\mathrm{dc}}^{*}\right)^{0} & \widetilde{i}_{L, 1}^{0} & \cdots & \widetilde{i}_{L, N}^{0}
\end{array}\right]^{T} .
\end{aligned}
$$

Note that the matrix $(\mathbf{J}-\mathbf{R})$ on the right-side of (A.24) is always nonsingular since it is negative definite. That is, $\widetilde{\mathbf{x}}^{T}(\mathbf{J}-$ $\mathbf{R}) \widetilde{\mathbf{x}}=\widetilde{\mathbf{x}}^{T} \mathbf{J} \widetilde{\mathbf{x}}-\widetilde{\mathbf{x}}^{T} \mathbf{R} \widetilde{\mathbf{x}}=-\widetilde{\mathbf{x}}^{T} \mathbf{J}^{T} \widetilde{\mathbf{x}}-\widetilde{\mathbf{x}}^{T} \mathbf{R} \widetilde{\mathbf{x}}=-\widetilde{\mathbf{x}}^{T} \mathbf{R} \widetilde{\mathbf{x}}<0, \forall \widetilde{\mathbf{x}} \neq \mathbf{0}$. Equation (A.24) indicates that $\widetilde{\mathbf{x}}=\mathbf{0}$, which shows that the following chain implications hold:

$$
\begin{aligned}
\left(\widetilde{v}_{\mathrm{dc}}^{*}\right)^{0} & =0, \\
\tilde{i}_{L, i}^{0} & =0, \quad i=1, \ldots, N \\
& \Downarrow \\
v_{\mathrm{dc}}^{0} & =\left(v_{\mathrm{dc}}^{*}\right)^{0}=v_{\mathrm{dc}, \mathrm{ref}}^{0} .
\end{aligned}
$$

Therefore, the verification of the result of (29) is completed.

\section{Conflicts of Interest}

The author declares that there are no conflicts of interest regarding the publication of this paper.

\section{Acknowledgments}

This research was supported by a grant from Transportation \& Logistics Research Program (TLRP) (17TLRP-C135446-01, Development of Hybrid Electric Vehicle Conversion Kit for Diesel Delivery Trucks and its Commercialization for Parcel Services) funded by Ministry of Land, Infrastructure and Transport of Korean Government.

\section{References}

[1] J. Moreno-Valenzuela and O. García-Alarcón, "On control of a boost DC-DC power converter under constrained input," Complexity, vol. 2017, Article ID 4143901, 2017.

[2] J. Huang, D. Xu, W. Yan, L. Ge, and X. Yuan, "Nonlinear Control of Back-to-Back VSC-HVDC System via CommandFilter Backstepping," Journal of Control Science and Engineering, vol. 2017, Article ID 7410392, 2017.

[3] N. Yang, C. Wu, R. Jia, and C. Liu, "Fractional-order terminal sliding-mode control for Buck DC/DC converter," Mathematical Problems in Engineering, vol. 2016, pp. 1-7, 2016.

[4] N. N. Yang, C. J. Wu, R. Jia, and C. Liu, "Modeling and characteristics analysis for a buck-boost converter in pseudocontinuous conduction mode based on fractional calculus," Mathematical Problems in Engineering, vol. 2016, Article ID 6835910, 11 pages, 2016. 
[5] S. K. Pidaparthy and B. Choi, "Control Design and Loop Gain Analysis of DC-to-DC Converters Intended for General Load Subsystems," Mathematical Problems in Engineering, vol. 2015, Article ID 426315, 2015.

[6] B. Salhi, H. El Fadil, T. Ahmed Ali, E. Magarotto, and F. Giri, "Adaptive output feedback control of interleaved parallel boost converters associated with fuel cell," Electric Power Components and Systems, vol. 43, no. 8-10, pp. 1141-1158, 2015.

[7] R. W. Erickson and D. Maksimović, Fundamentals of Power Electronics, Springer, Berlin, Germany, 2001.

[8] A. G. Perry, G. Feng, Y.-F. Liu, and P. C. Sen, "A design method for PI-like fuzzy logic controllers for DC-DC converter," IEEE Transactions on Industrial Electronics, vol. 54, no. 5, pp. 26882696, 2007.

[9] S. Bibian and H. Jin, "High performance predictive dead-beat digital controller for dc power supplies," IEEE Transactions on Power Electronics, vol. 17, no. 3, pp. 420-427, 2002.

[10] Q. Zhang, R. Min, Q. Tong, X. Zou, Z. Liu, and A. Shen, "Sensorless predictive current controlled DC-DC converter with a self-correction differential current observer," IEEE Transactions on Industrial Electronics, vol. 61, no. 12, pp. 6747-6757, 2014.

[11] M. Salimi, J. Soltani, A. Zakipour, and V. Hajbani, "Sliding mode control of the DC-DC flyback converter with zero steadystate error," in Proceedings of the 2013 4th Power Electronics, Drive Systems \& Technologies Conference (PEDSTC), pp. 158163, Tehran, Iran, Feburary 2013.

[12] J. Linares-Flores, A. Hernandez Mendez, C. Garcia-Rodriguez, and H. Sira-Ramirez, "Robust nonlinear adaptive control of a 'boost' converter via algebraic parameter identification," IEEE Transactions on Industrial Electronics, vol. 61, no. 8, pp. 41054114, 2014.

[13] A. G. Beccuti, S. Mariéthoz, S. Cliquennois, S. Wang, and M. Morari, "Explicit model predictive control of DC-DC switchedmode power supplies with extended kalman filtering," IEEE Transactions on Industrial Electronics, vol. 56, no. 6, pp. 18641874, 2009.

[14] Y.-X. Wang, D.-H. Yu, and Y.-B. Kim, "Robust time-delay control for the DC-DC boost converter," IEEE Transactions on Industrial Electronics, vol. 61, no. 9, pp. 4829-4837, 2014.

[15] W.-H. Chen, D. J. Ballance, P. J. Gawthrop, and J. O’Reilly, “A nonlinear disturbance observer for robotic manipulators," IEEE Transactions on Industrial Electronics, vol. 47, no. 4, pp. 932-938, 2000.

[16] Y. I. Son, I. H. Kim, D. S. Choi, and H. Shim, "Robust cascade control of electric motor drives using dual reduced-order PI observer," IEEE Transactions on Industrial Electronics, vol. 62, no. 6, pp. 3672-3682, 2015.

[17] S. Maniktala, Switching Power Supplies A-Z, Newnes, 2012.

[18] A. I. Pressman, "Switching Power Supply Design," in 1em plus 0.5em minus 0.4em, McGraw-Hill, New York, NY, USA, 1998.

[19] M. H. Rashid, Power Electronics, Pearson Prentice Hall, 3rd edition, 2004.

[20] H. K. Khalil, "Nonlinear Systems," in Nonlinear Systems, Prentice Hall, 2002. 


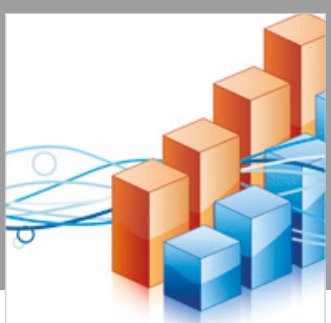

Advances in

Operations Research

\section{-n-m}
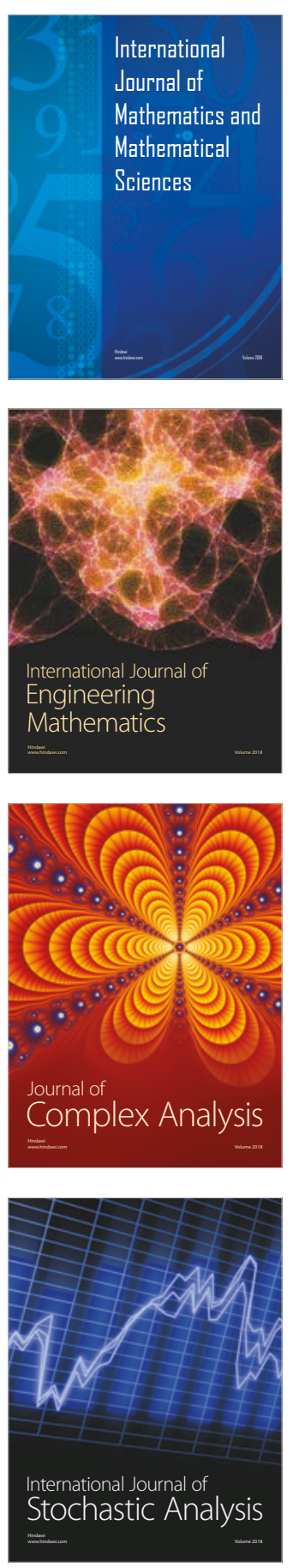
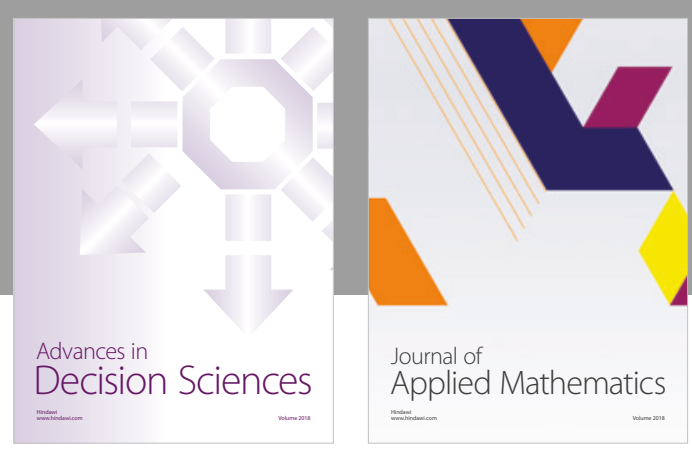

Journal of

Applied Mathematics
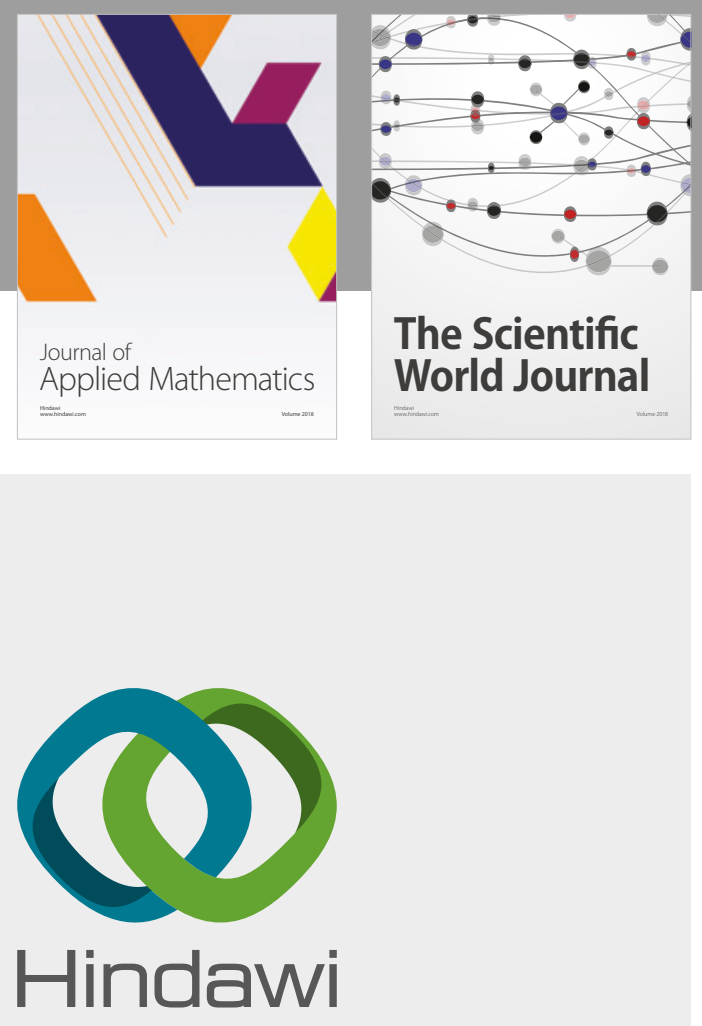

Submit your manuscripts at

www.hindawi.com

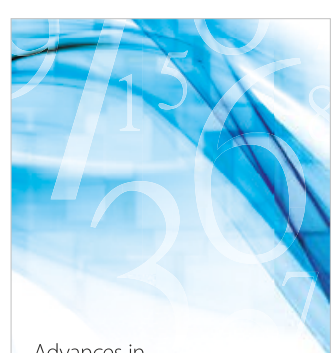

Advances in
Numerical Analysis
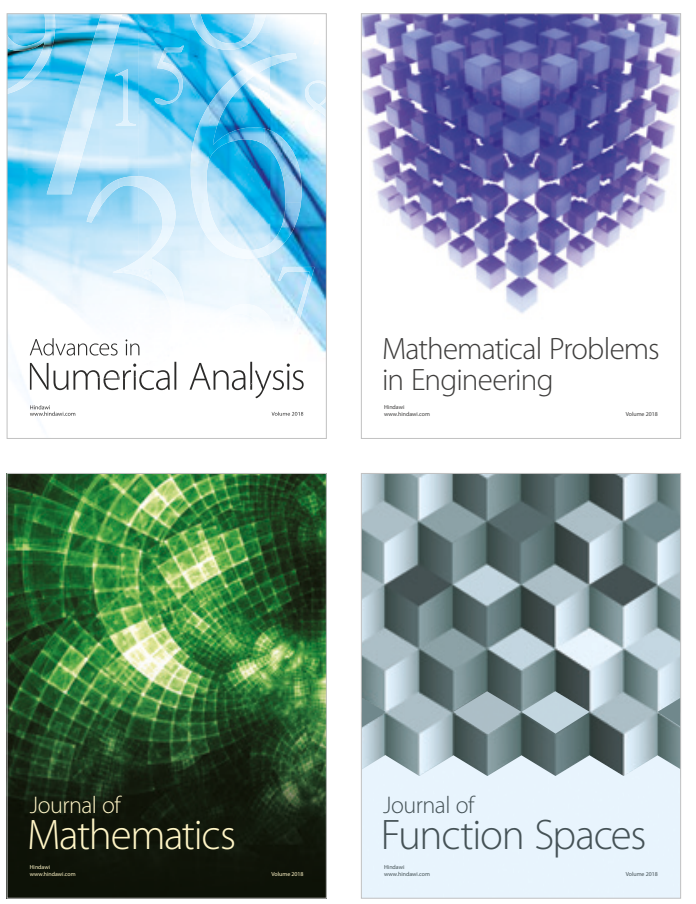

Mathematical Problems in Engineering

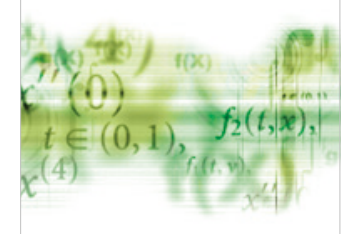

International Journal of

Differential Equations

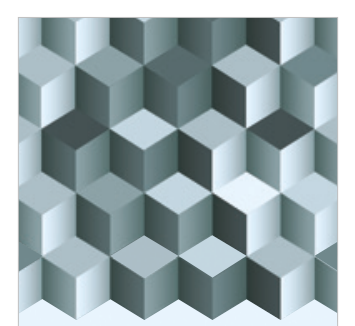

Journal of

Function Spaces

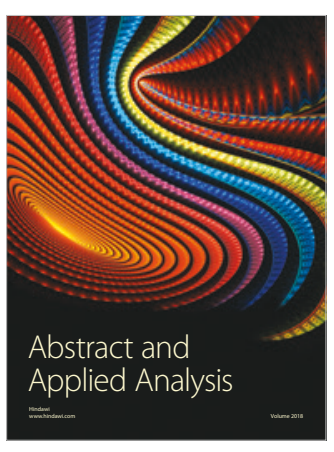

The Scientific

World Journal

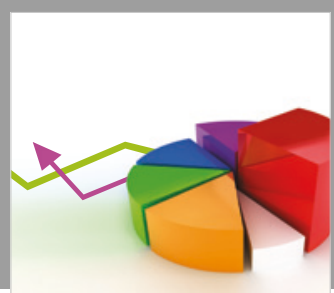

Journal of

Probability and Statistics
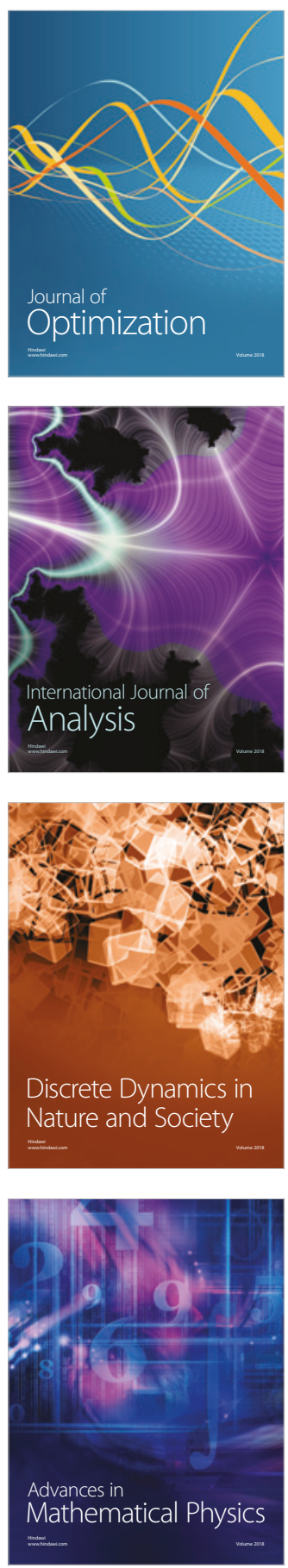